PROCEEDINGS OF THE

AMERICAN MATHEMATICAL SOCIETY

Volume 134, Number 1, Pages 303-310

S 0002-9939(05)07971-2

Article electronically published on June 13, 2005

\title{
THE POWER OF THE TANGENT BUNDLE OF THE REAL PROJECTIVE SPACE, ITS COMPLEXIFICATION AND EXTENDIBILITY
}

\author{
TEIICHI KOBAYASHI, HIRONORI YAMASAKI, AND TOSHIO YOSHIDA \\ (Communicated by Paul Goerss)
}

\begin{abstract}
We establish the formulas on the power $\tau^{k}$ of the tangent bundle $\tau=\tau\left(R P^{n}\right)$ of the real projective $n$-space $R P^{n}$ and its complexification $c \tau^{k}$, and apply the formulas to the problem of extendibility and stable extendiblity of $\tau^{k}$ and $c \tau^{k}$.
\end{abstract}

\section{INTRODUCTION}

Let $F$ denote either the real number field $R$ or the complex number field $C$, and let $X$ be a space and $A$ its subspace. A $t$-dimensional $F$-vector bundle $\zeta$ over $A$ is said to be extendible (respectively stably extendible) to $X$, if and only if there is a $t$-dimensional $F$-vector bundle over $X$ whose restriction to $A$ is equivalent (respectively stably equivalent) to $\zeta$ as $F$-vector bundles (cf. [9] and [3]). For simplicity, we use the same letter for a vector bundle and its equivalence class.

Let $R P^{n}$ denote the real projective $n$-space $R P^{n}$ and let $\tau=\tau\left(R P^{n}\right)$ stand for the tangent bundle of $R P^{n}$. We study the question: Determine the dimension $n$ for which an $F$-vector bundle over $R P^{n}$ is extendible (or stably extendible) to $R P^{m}$ for every $m \geq n$. We have obtained the complete answer for the tangent bundle $\tau=\tau\left(R P^{n}\right)$ in [6] and [8], for the complexification $c \tau$ of $\tau$ in [5], for the square $\tau^{2}=\tau\left(R P^{n}\right) \otimes \tau\left(R P^{n}\right)$ in [4] and for the complexification $c \tau^{2}$ of $\tau^{2}$ in [4], where $\otimes$ denotes the tensor product. The results on $\tau$ and $\tau^{2}$ are as follows.

Theorem 1.1 ([6, Theorem 6.6] and [ 8 , Theorem 4.2]). The following three conditions are equivalent:

(i) $\tau$ is extendible to $R P^{m}$ for every $m \geq n$.

(ii) $\tau$ is stably extendible to $R P^{m}$ for every $m \geq n$.

(iii) $n=1,3$ or 7 .

Theorem 1.2 ([4, Theorem 4]). The following three conditions are equivalent:

(i) $\tau^{2}$ is extendible to $R P^{m}$ for every $m \geq n$.

(ii) $\tau^{2}$ is stably extendible to $R P^{m}$ for every $m \geq n$.

(iii) $1 \leq n \leq 16$.

Received by the editors June 1, 2004 and, in revised form, August 30, 2004.

2000 Mathematics Subject Classification. Primary 55R50; Secondary 55N15.

Key words and phrases. Vector bundle, tangent bundle, real projective space, extendibility, stable extendibility, $K O$-theory, $K$-theory.

(C)2005 American Mathematical Society Reverts to public domain 28 years from publication 
The first purpose of this paper is to obtain the complete answer for the $k$-fold power $\tau^{k}$. Let $\phi(n)$ be the number of integers $s$ such that $0<s \leq n$ and $s \equiv 0,1$, 2 or $4 \bmod 8$. Then we have

Theorem A. For the $k$-fold power $\tau^{k}=\tau\left(R P^{n}\right) \otimes \cdots \otimes \tau\left(R P^{n}\right)$ (k-fold) of the tangent bundle $\tau\left(R P^{n}\right)$, the following three conditions are equivalent:

(i) $\tau^{k}$ is extendible to $R P^{m}$ for every $m \geq n$.

(ii) $\tau^{k}$ is stably extendible to $R P^{m}$ for every $m \geq n$.

(iii) There is an integer a satisfying

$$
(n+2)^{k}-n^{k} \leq a 2^{\phi(n)+1} \leq(n+2)^{k}+n^{k} .
$$

If $k=1$, the condition (iii) is equivalent to the condition: $n=1,3$ or 7 , and if $k=2$, it is equivalent to the condition: $1 \leq n \leq 16$. (Note that $2^{\phi(n)}>n+1$ for $n \neq 1,3,7$, and that $2^{\phi(n)}>n^{2}+2 n+2$ for $n \geq 17$.) Hence Theorem $\mathrm{A}$ is a generalization of Theorems 1.1 and 1.2. The results on $c \tau$ and $c \tau^{2}$ are as follows.

Theorem 1.3 ([5, Theorem 1]). The following three conditions are equivalent:

(i) $c \tau$ is extendible to $R P^{m}$ for every $m \geq n$.

(ii) $c \tau$ is stably extendible to $R P^{m}$ for every $m \geq n$.

(iii) $1 \leq n \leq 5$ or $n=7$.

Theorem 1.4 ([4, Theorem 5]). The following three conditions are equivalent:

(i) $c \tau^{2}$ is extendible to $R P^{m}$ for every $m \geq n$.

(ii) $c \tau^{2}$ is stably extendible to $R P^{m}$ for every $m \geq n$.

(iii) $1 \leq n \leq 17$.

The second purpose of this paper is to obtain the complete answer for the complexification $c \tau^{k}$ of $\tau^{k}$. For a real number $x$, let $[x]$ be the largest integer $n$ with $n \leq x$. Then we have

Theorem B. For the complexification $c \tau^{k}=c\left(\tau\left(R P^{n}\right) \otimes \cdots \otimes \tau\left(R P^{n}\right)\right)$ of the $k$-fold power $\tau^{k}$, the following three conditions are equivalent:

(i) $c \tau^{k}$ is extendible to $R P^{m}$ for every $m \geq n$.

(ii) $c \tau^{k}$ is stably extendible to $R P^{m}$ for every $m \geq n$.

(iii) There is an integer $b$ satisfying

$$
(n+2)^{k}-n^{k} \leq b 2^{[n / 2]+1} \leq(n+2)^{k}+n^{k} .
$$

If $k=1$, condition (iii) is equivalent to the condition $1 \leq n \leq 5$ or $n=7$, and if $k=2$, it is equivalent to the condition $1 \leq n \leq 17$. (Note that $2^{[n / 2]}>n+1$ for $n=6$ or $n \geq 8$, and that $2^{[n / 2]}>n^{2}+2 n+2$ for $n \geq 18$.) Hence Theorem $\mathrm{B}$ is a generalization of Theorems 1.3 and 1.4 .

This paper is arranged as follows. In Section 2 we establish the formulas on the power $\tau^{k}=\tau\left(R P^{n}\right) \otimes \cdots \otimes \tau\left(R P^{n}\right)$ of the tangent bundle $\tau\left(R P^{n}\right)$ of the real projective $n$-space $R P^{n}$. In Section 3 we apply the results in Section 2 to the problem of extendibility and stable extendibility of the $k$-fold power $\tau^{k}$ and prove Theorem A by using Theorem 4.1 in [8]. In Section 4 we establish the formulas on the complexification $c \tau^{k}=c\left(\tau\left(R P^{n}\right) \otimes \cdots \otimes \tau\left(R P^{n}\right)\right)$ of $\tau^{k}$. In Section 5 we apply the results in Section 4 to the problem of extendibility and stable extendibility of $c \tau^{k}$ and prove Theorem B by using Theorem 2.1 in [8]. 


\section{2 . The $k$-FOLD POWER OF THE TANGENT BUNDLE OF $\mathbb{R P}^{n}$}

In this section we establish the formulas on the $k$-fold power of the tangent bundle $\tau=\tau\left(R P^{n}\right)$. Let $\xi_{n}$ denote the canonical line bundle over $R P^{n}$.

Lemma 2.1. Let $\tau^{k}=\tau\left(R P^{n}\right) \otimes \cdots \otimes \tau\left(R P^{n}\right)$ (k-fold) denote the $k$-fold power of the tangent bundle $\tau=\tau\left(R P^{n}\right)$. Then, for any positive integer $r$, the following hold in the Grothendick group $K O\left(R P^{n}\right)$ :

(1) $\tau^{2 r-1}=2^{-1}\left\{(n+2)^{2 r-1}+n^{2 r-1}\right\} \xi_{n}-2^{-1}\left\{(n+2)^{2 r-1}-n^{2 r-1}\right\}$,

(2) $\tau^{2 r}=-2^{-1}\left\{(n+2)^{2 r}-n^{2 r}\right\} \xi_{n}+2^{-1}\left\{(n+2)^{2 r}+n^{2 r}\right\}$.

Proof. It is well known that $\tau=(n+1) \xi_{n}-1$ in $K O\left(R P^{n}\right)$. Hence formula (1) clearly holds for $r=1$.

Assume that formula (1) holds for $r \geq 1$. Then

$$
\begin{aligned}
\tau^{2 r} & =\tau \otimes \tau^{2 r-1} \\
& =\left\{(n+1) \xi_{n}-1\right\}\left[2^{-1}\left\{(n+2)^{2 r-1}+n^{2 r-1}\right\} \xi_{n}-2^{-1}\left\{(n+2)^{2 r-1}-n^{2 r-1}\right\}\right] \\
& =-2^{-1}\left\{(n+2)^{2 r}-n^{2 r}\right\} \xi_{n}+2^{-1}\left\{(n+2)^{2 r}+n^{2 r}\right\},
\end{aligned}
$$

since $\xi_{n} \otimes \xi_{n}=1$. So formula (2) holds for $r \geq 1$.

Assume that formula (2) holds for $r \geq 1$. Then

$$
\begin{aligned}
\tau^{2 r+1} & =\tau \otimes \tau^{2 r} \\
& =\left\{(n+1) \xi_{n}-1\right\}\left[2^{-1}\left\{(n+2)^{2 r}+n^{2 r}\right\}-2^{-1}\left\{(n+2)^{2 r}-n^{2 r}\right\} \xi_{n}\right] \\
& =2^{-1}\left\{(n+2)^{2 r+1}+n^{2 r+1}\right\} \xi_{n}-2^{-1}\left\{(n+2)^{2 r+1}-n^{2 r+1}\right\},
\end{aligned}
$$

since $\xi_{n} \otimes \xi_{n}=1$. So formula (1) holds for $r+1$.

Hence formulas (1) and (2) hold for any positive integer $r$ by induction on $r$.

The following result is used in our proofs.

Theorem 2.2 (cf. 2, Theorem 1.5, p. 100]). Two t-dimensional F-vector bundles over an $n$-dimensional $C W$-complex which are stably equivalent are equivalent if $\langle((n+2) / f)-1\rangle \leq t$, where $\langle x\rangle$ denotes the smallest integer $n$ with $x \leq n$ and $f=1$ or 2 according as $F=R$ or $C$.

We establish the formula on $\tau^{k}$, as follows.

Theorem 2.3. Let $\tau^{k}=\tau\left(R P^{n}\right) \otimes \cdots \otimes \tau\left(R P^{n}\right)$ ( $k$-fold) denote the $k$-fold power of the tangent bundle $\tau=\tau\left(R P^{n}\right)$. Then, for any positive integer $r$, the following hold:

(1) $\tau^{2 r-1} \oplus 2^{-1}\left\{(n+2)^{2 r-1}-n^{2 r-1}\right\}=2^{-1}\left\{(n+2)^{2 r-1}+n^{2 r-1}\right\} \xi_{n}$,

(2) $\tau^{2 r} \oplus 2^{-1}\left\{(n+2)^{2 r}-n^{2 r}\right\} \xi_{n}=2^{-1}\left\{(n+2)^{2 r}+n^{2 r}\right\}$,

where, in the equalities (1) and (2), a positive integer $k$ denotes the $k$-dimensional trivial bundle over $R P^{n}$ and $\oplus$ denotes the Whitney sum.

Proof. (1) By Lemma 2.1(1), we have

$$
\tau^{2 r-1}+2^{-1}\left\{(n+2)^{2 r-1}-n^{2 r-1}\right\}=2^{-1}\left\{(n+2)^{2 r-1}+n^{2 r-1}\right\} \xi_{n}
$$

in $K O\left(R P^{n}\right)$. Since

$$
\begin{aligned}
& \operatorname{dim}\left[\tau^{2 r-1} \oplus 2^{-1}\left\{(n+2)^{2 r-1}-n^{2 r-1}\right\}\right] \\
& \quad=2^{-1}\left\{(n+2)^{2 r-1}+n^{2 r-1}\right\}>n=\operatorname{dim} R P^{n}
\end{aligned}
$$


the equality

$$
\tau^{2 r-1} \oplus 2^{-1}\left\{(n+2)^{2 r-1}-n^{2 r-1}\right\}=2^{-1}\left\{(n+2)^{2 r-1}+n^{2 r-1}\right\} \xi_{n}
$$

holds as $R$-vector bundles by Theorem 2.2 .

(2) By Lemma 2.1(2), we have

$$
\tau^{2 r}+2^{-1}\left\{(n+2)^{2 r}-n^{2 r}\right\} \xi_{n}=2^{-1}\left\{(n+2)^{2 r}+n^{2 r}\right\}
$$

in $K O\left(R P^{n}\right)$. Since $\operatorname{dim}\left[\tau^{2 r} \oplus 2^{-1}\left\{(n+2)^{2 r}-n^{2 r}\right\} \xi_{n}\right]=2^{-1}\left\{(n+2)^{2 r}+n^{2 r}\right\}>$ $n=\operatorname{dim} R P^{n}$, the equality

$$
\tau^{2 r} \oplus 2^{-1}\left\{(n+2)^{2 r}-n^{2 r}\right\} \xi_{n}=2^{-1}\left\{(n+2)^{2 r}+n^{2 r}\right\}
$$

holds as $R$-vector bundles by Theorem 2.2

Moreover, the next theorem follows from Lemma 2.1

Theorem 2.4. For any positive integer $r$ and any integer a, the following hold in $K O\left(R P^{n}\right)$ :

(1) $\tau^{2 r-1}=2^{-1}\left\{(n+2)^{2 r-1}+n^{2 r-1}-a 2^{\phi(n)+1}\right\} \xi_{n}$ $+2^{-1}\left\{a 2^{\phi(n)+1}-(n+2)^{2 r-1}+n^{2 r-1}\right\}$,

(2) $\tau^{2 r}=2^{-1}\left\{a 2^{\phi(n)+1}-(n+2)^{2 r}+n^{2 r}\right\} \xi_{n}+2^{-1}\left\{(n+2)^{2 r}+n^{2 r}-a 2^{\phi(n)+1}\right\}$.

Proof. Subtracting $a 2^{\phi(n)}\left(\xi_{n}-1\right)=0$ (cf. [1, Theorem 7.4]) from equality (1) in Lemma 2.1 we have equality (1) above, and adding $a 2^{\phi(n)}\left(\xi_{n}-1\right)=0$ to equality (2) in Lemma 2.1] we have the equality (2) above.

\section{Extendibility AND StABle EXtendibility of the $k$-FOLD POWER

$$
\tau^{k}=\tau\left(\mathbb{R} \mathbb{P}^{n}\right) \otimes \cdots \otimes \tau\left(\mathbb{R} \mathbb{P}^{n}\right)
$$

Theorem 3.1. Assume that there is an integer a satisfying

$$
(n+2)^{k}-n^{k} \leq a 2^{\phi(n)+1} \leq(n+2)^{k}+n^{k} .
$$

Then $\tau^{k}$ is extendible to $R P^{m}$ for every $m \geq n$.

Proof. If $k=1$, the inequalities imply $a=1$ and $n=1,3$ or 7 , and if $n=1,3$ or 7, $\tau\left(R P^{n}\right)$ is trivial. Hence the results clearly hold for $n=1$ or $k=1$. So we may restrict our attention to the case $n>1$ and $k>1$.

In case $k$ is odd, let $k=2 r-1$, where $r$ is an integer $>1$. Then, by the assumption, we have

$$
2^{-1}\left\{(n+2)^{2 r-1}+n^{2 r-1}-a 2^{\phi(n)+1}\right\} \geq 0
$$

and

$$
2^{-1}\left\{a 2^{\phi(n)+1}-(n+2)^{2 r-1}+n^{2 r-1}\right\} \geq 0 .
$$

Hence Theorem 2.4(1) implies that the equality

$\tau^{2 r-1}=2^{-1}\left\{(n+2)^{2 r-1}+n^{2 r-1}-a 2^{\phi(n)+1}\right\} \xi_{n} \oplus 2^{-1}\left\{a 2^{\phi(n)+1}-(n+2)^{2 r-1}+n^{2 r-1}\right\}$ holds by Theorem 2.2 since $\operatorname{dim} \tau^{2 r-1}=n^{2 r-1}>n=\operatorname{dim} R P^{n}$ for $n>1$ and $r>1$. So $\tau^{2 r-1}$ is extendible to $R P^{m}$ for every $m \geq n$, since $\xi_{n}$ and the trivial bundle over $R P^{n}$ are extendible to $R P^{m}$ for every $m \geq n$.

In case $k$ is even, let $k=2 r$, where $r$ is a positive integer. Then, by the assumption, we have

$$
2^{-1}\left\{a 2^{\phi(n)+1}-(n+2)^{2 r}+n^{2 r}\right\} \geq 0
$$


and

$$
2^{-1}\left\{(n+2)^{2 r}+n^{2 r}-a 2^{\phi(n)+1}\right\} \geq 0 .
$$

Hence Theorem 2.4 (2) implies that the equality

$$
\tau^{2 r}=2^{-1}\left\{a 2^{\phi(n)+1}-(n+2)^{2 r}+n^{2 r}\right\} \xi_{n} \oplus 2^{-1}\left\{(n+2)^{2 r}+n^{2 r}-a 2^{\phi(n)+1}\right\}
$$

holds by Theorem 2.2, since $\operatorname{dim} \tau^{2 r}=n^{2 r}>n=\operatorname{dim} R P^{n}$ for $n>1$ and $r>0$. So $\tau^{2 r}$ is extendible to $R P^{m}$ for every $m \geq n$.

The following result is Theorem 4.1 in $[8]$ which is the stably extendible version of Theorem 6.2 in [6].

Theorem 3.2. Let $\zeta$ be a $t$-dimensional $R$-vector bundle over $R P^{n}$. Assume that there is a positive integer $l$ such that $\zeta$ is stably equivalent to $(t+l) \xi_{n}$, and $t+l<$ $2^{\phi(n)}$. Then $n<t+l$ and $\zeta$ is not stably extendible to $R P^{m}$ for every $m \geq t+l$.

Theorem 3.3. Assume that there is an integer a satisfying

$$
(n+2)^{k}+n^{k}-2^{\phi(n)+1}<a 2^{\phi(n)+1}<(n+2)^{k}-n^{k} .
$$

Then $\tau^{k}$ is not stably extendible to $R P^{m}$ for every $m \geq 2^{-1}\left\{(n+2)^{k}+n^{k}-a 2^{\phi(n)+1}\right\}$ if $k$ is odd, and for every $m \geq 2^{-1}\left\{(a+1) 2^{\phi(n)+1}-(n+2)^{k}+n^{k}\right\}$ if $k$ is even.

Proof. If $k$ is odd, let $k=2 r-1$. Then putting

$$
\zeta=\tau^{2 r-1}, \quad t=n^{2 r-1} \quad \text { and } \quad l=2^{-1}\left\{(n+2)^{2 r-1}-n^{2 r-1}-a 2^{\phi(n)+1}\right\}
$$

in Theorem 3.2, we obtain the result by Theorem 2.4(1) and Theorem 3.2, since $t+l<2^{\phi(n)}$ and $l>0$ by the assumption.

If $k$ is even, let $k=2 r$. Then putting

$$
\zeta=\tau^{2 r}, \quad t=n^{2 r} \quad \text { and } \quad l=2^{-1}\left\{(a+1) 2^{\phi(n)+1}-(n+2)^{2 r}-n^{2 r}\right\}
$$

in Theorem 3.2 we obtain the result by Theorem 2.4(2) and Theorem 3.2, since $t+l<2^{\phi(n)}$ and $l>0$ by the assumption.

Proof of Theorem A. (i) clearly implies (ii). (iii) implies (i) by Theorem 3.1. To show that (ii) implies (iii), we prove the contraposition. Assume that every integer $a$ satisfies

$$
a 2^{\phi(n)+1}<(n+2)^{k}-n^{k} \text { or }(n+2)^{k}+n^{k}<a 2^{\phi(n)+1} .
$$

Assume that there are integers $a$ with $a 2^{\phi(n)+1}<(n+2)^{k}-n^{k}$. Then we define $A$ as the maximum integer such that $A 2^{\phi(n)+1}<(n+2)^{k}-n^{k}$. If $A$ satisfies $A 2^{\phi(n)+1} \leq(n+2)^{k}+n^{k}-2^{\phi(n)+1}$, we have $(n+2)^{k}-n^{k} \leq(A+1) 2^{\phi(n)+1} \leq$ $(n+2)^{k}+n^{k}$, and these are inconsistent with our assumption. Hence $A$ satisfies $(n+2)^{k}+n^{k}-2^{\phi(n)+1}<A 2^{\phi(n)+1}<(n+2)^{k}-n^{k}$. So, by Theorem 3.3 $\tau^{k}$ is not stably extendible to $R P^{m}$ for every $m \geq 2^{-1}\left\{(n+2)^{k}+n^{k}-A 2^{\phi(n)+1}\right\}$ if $k$ is odd, and for every $m \geq 2^{-1}\left\{(A+1) 2^{\phi(n)+1}-(n+2)^{k}+n^{k}\right\}$ if $k$ is even.

Assume that there are integers $a$ with $(n+2)^{k}+n^{k}<a 2^{\phi(n)+1}$. Then we define $B$ as the minimum integer such that $(n+2)^{k}+n^{k}<B 2^{\phi(n)+1}$. If $B$ satisfies $B 2^{\phi(n)+1} \geq(n+2)^{k}-n^{k}+2^{\phi(n)+1}$, we have $(n+2)^{k}-n^{k} \leq(B-1) 2^{\phi(n)+1} \leq$ $(n+2)^{k}+n^{k}$, and these are inconsistent with our assumption. Hence $B$ satisfies $(n+2)^{k}+n^{k}-2^{\phi(n)+1}<(B-1) 2^{\phi(n)+1}<(n+2)^{k}-n^{k}$. So, by Theorem 3.3 $\tau^{k}$ is not stably extendible to $R P^{m}$ for every $m \geq 2^{-1}\left\{(n+2)^{k}+n^{k}-(B-1) 2^{\phi(n)+1}\right\}$ if $k$ is odd, and for every $m \geq 2^{-1}\left\{B 2^{\phi(n)+1}-(n+2)^{k}+n^{k}\right\}$ if $k$ is even. 


\section{The COMPlexificAtion of The $k$-FOLD POWER OF $\tau\left(\mathbb{R} \mathbb{P}^{n}\right)$}

Complexifying the equalities (1) and (2) in Lemma 2.1] we immediately have

Lemma 4.1. Let $c \tau^{k}=c\left(\tau\left(R P^{n}\right) \otimes \cdots \otimes \tau\left(R P^{n}\right)\right)$ denote the complexification of the $k$-fold power $\tau^{k}$ of the tangent bundle $\tau=\tau\left(R P^{n}\right)$. Then, for any positive integer $r$, the following hold in the Grothendick group $K\left(R P^{n}\right)$ :

(1) $c \tau^{2 r-1}=2^{-1}\left\{(n+2)^{2 r-1}+n^{2 r-1}\right\} c \xi_{n}-2^{-1}\left\{(n+2)^{2 r-1}-n^{2 r-1}\right\}$,

(2) $c \tau^{2 r}=-2^{-1}\left\{(n+2)^{2 r}-n^{2 r}\right\} c \xi_{n}+2^{-1}\left\{(n+2)^{2 r}+n^{2 r}\right\}$.

Complexifying the equalities (1) and (2) in Theorem 2.3, we immediately have

Theorem 4.2. For the complexification $c \tau^{k}=c\left(\tau\left(R P^{n}\right) \otimes \cdots \otimes \tau\left(R P^{n}\right)\right)$ of the $k$-fold power $\tau^{k}$ of the tangent bundle $\tau=\tau\left(R P^{n}\right)$, the following hold:

(1) $c \tau^{2 r-1} \oplus 2^{-1}\left\{(n+2)^{2 r-1}-n^{2 r-1}\right\}=2^{-1}\left\{(n+2)^{2 r-1}+n^{2 r-1}\right\} c \xi_{n}$,

(2) $c \tau^{2 r} \oplus 2^{-1}\left\{(n+2)^{2 r}-n^{2 r}\right\} c \xi_{n}=2^{-1}\left\{(n+2)^{2 r}+n^{2 r}\right\}$.

Furthermore, the next theorem follows from Lemma 4.1

Theorem 4.3. For any positive integer $r$ and any integer $b$, the following hold in $K\left(R P^{n}\right)$ :

(1) $c \tau^{2 r-1}=2^{-1}\left\{(n+2)^{2 r-1}+n^{2 r-1}-b 2^{[n / 2]+1}\right\} c \xi_{n}$ $+2^{-1}\left\{b 2^{[n / 2]+1}-(n+2)^{2 r-1}+n^{2 r-1}\right\}$,

(2) $c \tau^{2 r}=2^{-1}\left\{b 2^{[n / 2]+1}-(n+2)^{2 r}+n^{2 r}\right\} c \xi_{n}+2^{-1}\left\{(n+2)^{2 r}+n^{2 r}-b 2^{[n / 2]+1}\right\}$.

Proof. Subtracting $b 2^{[n / 2]}\left(\xi_{n}-1\right)=0$ (cf. [1, Theorem 7.3]) from equality (1) in Lemma 4.1, we have equality (1) above, and adding $b 2^{[n / 2]}\left(\xi_{n}-1\right)=0$ to equality (2) in Lemma 4.1, we have equality (2) above.

\section{EXTENDIBILITY AND STABLE EXTENDIBILITY OF THE COMPLEXIFICATION $c \tau^{k}=c\left(\tau\left(\mathbb{R} \mathbb{P}^{n}\right) \otimes \cdots \otimes \tau\left(\mathbb{R P}^{n}\right)\right)$}

The proofs of the following Theorems 5.1 and 5.3 are parallel to those of Theorems 3.1 and 3.3 , respectively.

Theorem 5.1. Assume that there is an integer $b$ satisfying

$$
(n+2)^{k}-n^{k} \leq b 2^{[n / 2]+1} \leq(n+2)^{k}+n^{k} .
$$

Then $c \tau^{k}$ is extendible to $R P^{m}$ for every $m \geq n$.

Proof. In case $k$ is odd, let $k=2 r-1$, where $r$ is a positive integer. Then, by the assumption, we have

$$
2^{-1}\left\{(n+2)^{2 r-1}+n^{2 r-1}-b 2^{[n / 2]+1}\right\} \geq 0
$$

and

$$
2^{-1}\left\{b 2^{[n / 2]+1}-(n+2)^{2 r-1}+n^{2 r-1}\right\} \geq 0 .
$$

Hence Theorem 4.3(1) implies that the equality

$c \tau^{2 r-1}=2^{-1}\left\{(n+2)^{2 r-1}+n^{2 r-1}-b 2^{[n / 2]+1}\right\} c \xi_{n} \oplus 2^{-1}\left\{b 2^{[n / 2]+1}-(n+2)^{2 r-1}+n^{2 r-1}\right\}$ holds by Theorem 2.2 , since $\operatorname{dim} c \tau^{2 r-1}=n^{2 r-1} \geq\langle(n+2) / 2-1\rangle=\langle n / 2\rangle$. So $c \tau^{2 r-1}$ is extendible to $R P^{m}$ for every $m \geq n$, since $c \xi_{n}$ and the trivial bundle over $R P^{n}$ are extendible to $R P^{m}$ for every $m \geq n$. 
In case $k$ is even, let $k=2 r$, where $r$ is a positive integer. Then, by the assumption, we have

$$
2^{-1}\left\{b 2^{[n / 2]+1}-(n+2)^{2 r}+n^{2 r}\right\} \geq 0
$$

and

$$
2^{-1}\left\{(n+2)^{2 r}+n^{2 r}-b 2^{[n / 2]+1}\right\} \geq 0 .
$$

Hence Theorem 4.3(2) implies that the equality

$$
c \tau^{2 r}=2^{-1}\left\{b 2^{[n / 2]+1}-(n+2)^{2 r}+n^{2 r}\right\} c \xi_{n} \oplus 2^{-1}\left\{(n+2)^{2 r}+n^{2 r}-b 2^{[n / 2]+1}\right\}
$$

holds by Theorem 2.2, since $\operatorname{dim} c \tau^{2 r}=n^{2 r} \geq\langle(n+2) / 2-1\rangle=\langle n / 2\rangle$. So $c \tau^{2 r}$ is extendible to $R P^{m}$ for every $m \geq n$.

The following result is Theorem 2.1 in 8 , which is the stably extendible version of Theorem 4.2 for $d=1$ in [7].

Theorem 5.2. Let $\zeta$ be a t-dimensional $C$-vector bundle over $R P^{n}$. Assume that there is a positive integer $l$ such that $\zeta$ is stably equivalent to $(t+l) c \xi_{n}$, and $t+l<$ $2^{[n / 2]}$. Then $n<2 t+2 l$ and $\zeta$ is not stably extendible to $R P^{m}$ for every $m \geq 2 t+2 l$.

Theorem 5.3. Assume that there is an integer $b$ satisfying

$$
(n+2)^{k}+n^{k}-2^{[n / 2]+1}<b 2^{[n / 2]+1}<(n+2)^{k}-n^{k} .
$$

Then $c \tau^{k}$ is not stably extendible to $R P^{m}$ for every $m \geq(n+2)^{k}+n^{k}-b 2^{[n / 2]+1}$ if $k$ is odd, and for every $m \geq(b+1) 2^{[n / 2]+1}-(n+2)^{k}+n^{k}$ if $k$ is even.

Proof. If $k$ is odd, let $k=2 r-1$. Then putting

$$
\zeta=c \tau^{2 r-1}, \quad t=n^{2 r-1} \quad \text { and } \quad l=2^{-1}\left\{(n+2)^{2 r-1}-n^{2 r-1}-b 2^{[n / 2]+1}\right\}
$$

in Theorem 5.2, we obtain the result by Theorem 4.3(1) and Theorem 5.2, since $t+l<2^{[n / 2]}$ and $l>0$ by the assumption.

If $k$ is even, let $k=2 r$. Then putting

$$
\zeta=c \tau^{2 r}, \quad t=n^{2 r} \quad \text { and } \quad l=2^{-1}\left\{(b+1) 2^{[n / 2]+1}-(n+2)^{2 r}-n^{2 r}\right\}
$$

in Theorem [5.2] we obtain the result by Theorem 4.3(2) and Theorem [5.2, since $t+l<2^{[n / 2]}$ and $l>0$ by the assumption.

Proof of Theorem B. (i) implies (ii) clearly. (iii) implies (i) by Theorem 5.1. To show that (ii) implies (iii), we prove the contraposition. Assume that every integer $b$ satisfies

$$
b 2^{[n / 2]+1}<(n+2)^{k}-n^{k} \text { or }(n+2)^{k}+n^{k}<b 2^{[n / 2]+1} .
$$

Assume that there are integers $b$ with $b 2^{[n / 2]+1}<(n+2)^{k}-n^{k}$. Then we define $C$ as the maximum integer such that $C 2^{[n / 2]+1}<(n+2)^{k}-n^{k}$. If $C$ satisfies $C 2^{[n / 2]+1} \leq(n+2)^{k}+n^{k}-2^{[n / 2]+1}$, we have $(n+2)^{k}-n^{k} \leq(C+1) 2^{[n / 2]+1} \leq$ $(n+2)^{k}+n^{k}$, and these are inconsistent with our assumption. Hence $C$ satisfies $(n+2)^{k}+n^{k}-2^{[n / 2]+1}<C 2^{[n / 2]+1}<(n+2)^{k}-n^{k}$. So, by Theorem [5.3 $c \tau^{k}$ is not stably extendible to $R P^{m}$ for every $m \geq(n+2)^{k}+n^{k}-C 2^{[n / 2]+1}$ if $k$ is odd, and for every $m \geq(C+1) 2^{[n / 2]+1}-(n+2)^{k}+n^{k}$ if $k$ is even.

Assume that there are integers $b$ with $(n+2)^{k}+n^{k}<b 2^{[n / 2]+1}$. Then we define $D$ as the minimum integer such that $(n+2)^{k}+n^{k}<D 2^{[n / 2]+1}$. If $D$ satisfies $D 2^{[n / 2]+1} \geq(n+2)^{k}-n^{k}+2^{[n / 2]+1}$, we have $(n+2)^{k}-n^{k} \leq(D-1) 2^{[n / 2]+1} \leq$ $(n+2)^{k}+n^{k}$, and these are inconsistent with our assumption. Hence $D$ satisfies 
$(n+2)^{k}+n^{k}-2^{[n / 2]+1}<(D-1) 2^{[n / 2]+1}<(n+2)^{k}-n^{k}$. So, by Theorem [5.3. $c \tau^{k}$ is not stably extendible to $R P^{m}$ for every $m \geq(n+2)^{k}+n^{k}-(D-1) 2^{[n / 2]+1}$ if $k$ is odd, and for every $m \geq D 2^{[n / 2]+1}-(n+2)^{k}+n^{k}$ if $k$ is even.

\section{REFERENCES}

1. J. F. Adams, Vector fields on spheres, Ann. of Math. 75 (1962), 603-632. MR0139178 $(25: 2614)$

2. D. Husemoller, Fibre bundles, Second edition, Graduate Texts in Mathmatics 20, SpringerVerlag, New York-Hiderberg-Berlin, 1975. MR0370578 (51:6805)

3. M. Imaoka and K. Kuwana, Stably extendible vector bundles over the quaternionic projective spaces, Hiroshima Math. J. 29 (1999), 273-279. MR1704248(2000h:55027)

4. T. Kobayashi and K. Komatsu, Extendiblity and stable extendibility of vector bundles over real projective spaces, Hiroshima Math. J. 31 (2001), 99-106. MR1820697 (2001j:55018)

5. T. Kobayashi and T. Yoshida, Extendible and stably extendible vector bundles over real projective spaces, J. Math. Soc. Japan 55 (2003), 1053-1059. MR2003759 (2004i:55022)

6. T. Kobayashi, H. Maki and T. Yoshida, Remarks on extendible vector bundles over lens spaces and real projective spaces, Hiroshima Math. J. 5 (1975), 487-497. MR 0385857(52:6716)

7. T. Kobayashi, H. Maki and T. Yoshida, Extendiblity with degree $d$ of the complex vector bundles over lens spaces and projective spaces, Mem. Fac. Sci Kochi Univ. (Math.) 1 (1980), 23-33. MR 0561386 (81c:55027)

8. T. Kobayashi, H. Maki and T. Yoshida, Stably extendible vector bundles over the real projective spaces and the lens spaces, Hiroshima Math. J. 29 (1999), 631-638. MR.1728614|(2000k:55023)

9. R. L. E. Schwarzenberger, Extendible vector bundles over real projective space, Quart. J. Math. Oxford (2) 17 (1966), 19-21. MR0195105 (33:3310)

Department of Mathematics, Faculty of Science, Kochi University, Kochi 780-8520, JAPAN

E-mail address: kteiichi@lime.ocn.ne.jp

Department of Mathematics, Graduate School of Science, Hiroshima University, HigASHI-HiRoshima 739-8526, JAPAN

E-mail address: d042710@math.sci.hiroshima-u.ac.jp

Department of Mathematics, Faculty of Integrated Arts and Sciences, Hiroshima University, Higashi-Hiroshima 739-8521, JaPAN

E-mail address: t-yosida@mis.hiroshima-u.ac.jp 